\title{
A method to multi-attribute decision making with picture fuzzy information based on Muirhead mean
}

Article

Accepted Version

Xu, Y., Shang, X., Wang, J., Zhang, R., Li, W. and Xing, Y. (2019) A method to multi-attribute decision making with picture fuzzy information based on Muirhead mean. Journal of Intelligent and Fuzzy Systems, 36 (4). pp. 3833-3849. ISSN 1875-8967 doi: https://doi.org/10.3233/JIFS-172130 Available at https://centaur.reading.ac.uk/78223/

It is advisable to refer to the publisher's version if you intend to cite from the work. See Guidance on citing.

To link to this article DOI: http://dx.doi.org/10.3233/JIFS-172130

Publisher: IOS Press

All outputs in CentAUR are protected by Intellectual Property Rights law, including copyright law. Copyright and IPR is retained by the creators or other copyright holders. Terms and conditions for use of this material are defined in the End User Agreement.

www.reading.ac.uk/centaur 
Central Archive at the University of Reading

Reading's research outputs online 


\title{
A method to multi-attribute decision making with picture fuzzy information based on Muirhead mean
}

\author{
Yuan Xu, Xiaopu Shang*, Jun Wang, Runtong Zhang, Weizi Li and Yuping Xing \\ School of Economics and Management, Beijing Jiaotong University, Beijing, China \\ Informatics Research Centre, Henley Busienss School, University of Reading, UK
}

\begin{abstract}
The recently proposed picture fuzzy set (PFS) is a powerful tool for handling fuzziness and uncertainty. PFS is characterized by a positive membership degree, a neutral membership degree, and a negative membership degree, making it more suitable and useful than the intuitionistic fuzzy set (IFS) when dealing with multi-attribute decision making (MADM). The aim of this paper is to develop some aggregation operators for fusing picture fuzzy information. Considering the Muirhead mean $(\mathrm{MM})$ is an aggregation technology which can consider the interrelationship among all aggregated arguments, we extend MM to picture fuzzy context and propose a family of picture fuzzy Muirhead mean operators. In addition, we investigate some properties and special cases of the proposed operators. Further, we develop a novel method to MADM in which the attribute values take the form of picture fuzzy numbers (PFNs). Finally, a numerical example is provided to illustrate the validity of the proposed method.
\end{abstract}

Keywords: picture fuzzy set, multi-attribute decision making, aggregation operators, Muirhead mean

\section{Introduction}

Due to the complexity in real decision-making problems, we always face the difficulties of presenting attribute values correctly and appropriately. Actually, as fuzziness and uncertainty do exist in real MADM problems, we should consider the issue of dealing with both. Recently, more and more scholars have paid their attention to tools that can effectively handle vagueness and impreciseness. Zadeh's [1] fuzzy set (FS) theory is primary technology for dealing with ambiguity. On the basis of Zadeh's pioneering works, decision making with fuzzy information have been widely investigated [2-4].Thereafter, Atanassov [5] extended FS and proposed IFS, constructed by a membership degree and a nonmembership degree. Evidently, IFS has higher capacity of modelling the fuzziness of information than FS .Since its introduction, it has received much attention and been widely investigated. For instance, $\mathrm{Xu}$ [6] extended the ordered weighted averaging operator to IFSs and developed a series of intuitionistic fuzzy aggregation operators. Qin and Liu [7] proposed some intuitionistic fuzzy Maclaurin symmetric mean and applied them to MADM. Guo [8] proposed a new risk assessment methodology which combines IFSs with evidence theory. Cao et al. [9] proposed an intuitionistic fuzzy multi-criteria decision-making method for supplier selection problem. Wan et al. [10] proposed a novel three-phase method for group decision with interval-valued intuitionistic fuzzy preference relations. Wang and Chen [11] proposed a new MADM method based on linear programming methodology in interval-valued intuitionistic fuzzy environment. In addition, quite a few intuitionistic fuzzy aggregation operators have been proposed [12-20]. Moreover, due to its advantages and merits, IFSs have also been applied in $[21,22]$, clustering analysis $[23,24]$, and pattern recognition [25, 26].

Although IFSs have been successfully applied in quite a few fields, there exist situations that cannot be represented by IFSs. For example, in the case of voting, human opinion involving more answers of types: yes, abstain, no, and refusal, that cannot be represented by IFSs. Thus, to deal with this situation, Coung [27] proposed the concept of PFS, which is 
characterized by three information functions, i.e. a positive membership degree, a neural membership degree and a negative membership degree. Compared with IFS, PFS is more powerful and suitable to cope with circumstances requiring human opinions involving more answers of types: yes, abstain, no, refusal. Due to this feature, it has drawn much attention since its introduction. For instance, Le et al [28] proposed a novel fuzzy inference system on PFSs called the picture fuzzy inference system to enhance inference performance of the traditional fuzzy inference system. Le and Pham [29] proposed two novel hybrid forecast methods based on the picture fuzzy clustering for weather nowcasting. Wei [30] proposed some novel similarity measures of PFSs and applied them for building material recognition and minerals field recognition. When dealing with MADM with picture fuzzy information, picture fuzzy aggregation operators are needed. Recently, some picture fuzzy operators have been developed. Garg [31] and Wei [32] proposed a family of picture fuzzy averaging and geometric operators respectively.

The above proposed operators can be successfully applied to aggregate picture fuzzy numbers. However, the main flaw of these operators is that none of them can consider the interrelationship among aggregated PFNs. In other words, these operators assume that attributes are independent, which is somewhat counterintuitive. In most situations, attributes are related so that not only the attribute values themselves but also the interrelationships among them should be taken into account when calculating overall assessment values of alternatives. Basically, Bonferroni mean (BM) [33] and the Heronian mean (HM) [34] are two powerful aggregation technologies, which can capture the interrelationship between arguments. Recently BM, HM and their extensions have been successfully applied to aggregating intuitionistic fuzzy information [35-38], dual hesitant fuzzy information $[39,40]$ and so forth. Nevertheless, both BM can only consider the interrelationship between any two arguments and fail to capture the interrelationship among multiple arguments. Maclaurin's [41] Maclaurin symmetric mean (MSM) is also an effective aggregation technology which considers the interrelationship among multiple arguments. However, MSM cannot capture the interrelationships among all arguments either.

Recently, Muirhead [42] introduced a powerful aggregation operator, called MM. MM is well known for its ability of reflecting the interrelationships among all input arguments. Moreover, MM has a vector of parameters, making the information aggregation process flexible. By assigning different values to the vector of parameters, different aggregation results can be derived. Additionally, some existing operators, such as BM and MSM are special cases of MM with respect to the vector of parameter. Recently, MM has been utilized to aggregate 2-tuple linguistic information [43], intuitionistic fuzzy information [44], Pythagorean fuzzy information [45], and hesitant fuzzy linguistic information [46, 47]. Nevertheless, to the best of our knowledge, nothing has been done about MM in PFSs. Therefore, to take advantages of MM, we utilize MM to aggregate PFNs and propose a family of picture fuzzy aggregation operators. Further, we propose a novel approach to MADM based on the proposed operators.

The main motivations and objectives of this are: (1) to propose new picture fuzzy aggregation operators based on MM; (2) to propose a novel approach to MADM in which attribute values take the form of PFNs. The rest of the paper is organized as follows. Section 2 recalls some relevant concepts. Section 3 proposes some picture fuzzy Muirhead mean operators with their properties. Some desirable properties and special cases are also studied. Section 4 introduces a novel method to MADM with picture fuzzy sets based on the proposed aggregation operators. Section 5 provides an example to illustrate the validity of the proposed method.

\section{Basic concepts}

This section briefly describes s some concepts about PFS and MM.

\subsection{Picture fuzzy set}

Definition 1. [27] Let $X$ be an ordinary fixed set, a PFS $A$ defined on $X$ is expressed as follows:

$$
A=\left\{\left\langle x, \mu_{A}(x), \eta_{A}(x), v_{A}(x)\right\rangle \mid x \in X\right\},
$$

where $\mu_{A}(x), \eta_{A}(x)$ and $v_{A}(x)$ denote the positive membership, neutral membership and negative membership degrees respectively, satisfying $\mu_{A}(x) \in[0,1]$, $\eta_{A}(x) \in[0,1] \quad, \quad v_{A}(x) \in[0,1] \quad$ and $0 \leq \mu_{A}(x)+\eta_{A}(x)+v_{A}(x) \leq 1$

Then $\pi_{A}(x)=1-\left(\mu_{A}(x)+\eta_{A}(x)+v_{A}(x)\right)$ is called as the refusal membership of $x$ in $A$. For convenience, $\alpha=\left(\mu_{\alpha}, \eta_{\alpha}, v_{\alpha}\right)$ is called as a PFN by Wei [32]. 
In addition, Wei [32] gave some operations for PFNs.

Definition 2. [32] Let $\alpha=\left(\mu_{\alpha}, \eta_{\alpha}, v_{\alpha}\right)$ and $\beta=\left(\mu_{\beta}, \eta_{\beta}, v_{\beta}\right)$ be two PFNs, $\lambda$ be a positive real number.

Then

(1) $\alpha \oplus \beta=\left(\mu_{\alpha}+\mu_{\beta}-\mu_{\alpha} \mu_{\beta}, \eta_{\alpha} \eta_{\beta}, v_{\alpha} v_{\beta}\right)$,

(2) $\alpha \otimes \beta=\left(\mu_{\alpha} \mu_{\beta}, \eta_{\alpha}+\eta_{\beta}-\eta_{\alpha} \eta_{\beta}, v_{\alpha}+v_{\beta}-v_{\alpha} v_{\beta}\right)$,

(3) $\lambda \alpha=\left(1-\left(1-\mu_{\alpha}\right)^{\lambda}, \eta_{\alpha}^{\lambda}, v_{\alpha}^{\lambda}\right)$,

(4) $\alpha^{\lambda}=\left(\mu_{\alpha}^{\lambda}, 1-\left(1-\eta_{\alpha}\right)^{\lambda}, 1-\left(1-v_{\alpha}\right)^{\lambda}\right)$.

To compare any two PFNs, Wei [32] developed a comparison rule for PFNs.

Definition 3 [32]. Let $\alpha=(\mu, \eta, v)$ be a PFN, then the score of $\alpha$ is defined as $S(\alpha)=\mu-v$, and the accuracy of $\alpha$ is defined as $H(\alpha)=\mu+\eta+v$. For any two FPNs, $\alpha_{1}=\left(\mu_{1}, \eta_{1}, v_{1}\right)$ and $\alpha_{2}=\left(\mu_{2}, \eta_{2}, v_{2}\right)$. Then

(1) If $S\left(\alpha_{1}\right)>S\left(\alpha_{2}\right)$, then $\alpha_{1}>\alpha_{2}$;

(2) If $S\left(\alpha_{1}\right)=S\left(\alpha_{2}\right)$, then

$$
\begin{aligned}
& \text { if } H\left(\alpha_{1}\right)>H\left(\alpha_{2}\right) \text {, then } \alpha_{1}>\alpha_{2} \text {; } \\
& \text { if } H\left(\alpha_{1}\right)=H\left(\alpha_{2}\right) \text {, then } \alpha_{1}=\alpha_{2} \text {. }
\end{aligned}
$$

\subsection{Muirhead mean}

The MM was proposed by Muirhead [42] for crisp numbers. The advantage of $\mathrm{MM}$ is that it can consider the interrelationships among all aggregated arguments.

Definition 4. [42] Let $a_{i}(i=1,2, \ldots, n)$ be a collection of crisp numbers and $P=\left(p_{1}, p_{2}, \ldots, p_{n}\right) \in R^{n}$ be a vector of parameters, then Muirhead mean (MM) operator is defined as

$$
M^{P}\left(a_{1}, a_{2}, \ldots, a_{n}\right)=\left(\frac{1}{n !} \sum_{\vartheta \in S_{n}} \prod_{j=1}^{n} a_{\vartheta(j)}^{p_{j}}\right)^{\frac{1}{\sum_{j=1}^{n} p_{j}}},
$$

where $\vartheta(j)(j=1,2, \ldots, n)$ is any permutation of $(1,2, \ldots, n)$, and $S_{n}$ is the collection of all permutation of $(1,2, \ldots, n)$.

Moreover, Liu and Li [44] proposed the dual Muirhead mean (DMM) operator.
Definition 5. [44] Let $a_{i}(i=1,2, \ldots, n)$ be a collection of crisp numbers and $P=\left(p_{1}, p_{2}, \ldots, p_{n}\right) \in R^{n}$ be a vector of parameters, then DMM operator is defined as

$$
\operatorname{DMM}^{P}\left(a_{1}, a_{2}, \ldots, a_{n}\right)=\frac{1}{\sum_{j=1}^{n} p_{j}}\left(\prod_{\vartheta \in S_{n}} \sum_{j=1}^{n}\left(p_{j} a_{\vartheta(j)}\right)\right)^{\frac{1}{n !}},
$$

where $\vartheta(j)(j=1,2, \ldots, n)$ is any permutation of $(1,2, \ldots, n)$, and $S_{n}$ is the collection of all permutations of $(1,2, \ldots, n)$.

\section{The picture fuzzy Muirhead mean operators}

In this section, we extend MM to picture fuzzy environment and propose a family of picture fuzzy Muirhead mean operators.

\subsection{The picture fuzzy Muirhead mean operator}

Definition 6. Let $\alpha_{j}=\left(\mu_{j}, \eta_{j}, v_{j}\right)(j=1,2, \mathrm{~K}, n)$ be a collection of PFNs, and $P=\left(p_{1}, p_{2}, \ldots, p_{n}\right) \in R^{n}$ be a vector of parameters, then the picture fuzzy Muirhead mean (PFMM) operator is defined as

$$
\operatorname{PFMM}^{p}\left(\alpha_{1}, \alpha_{2}, \mathrm{~K}, \alpha_{n}\right)=\left(\frac{1}{n !} \sum_{\vartheta \in S_{n}} \prod_{j=1}^{n} \alpha_{\vartheta(j)}^{p_{j}}\right)^{\frac{1}{\sum_{j=1}^{n} p_{j}}},
$$

where $\vartheta(j)(j=1,2, \ldots, n)$ is any permutation of $(1,2, \ldots, n)$, and is the collection of all permutations of $(1,2, \ldots, n)$.

According to Definition 2, the following theorem can be obtained.

Theorem 1. Let $\alpha_{j}=\left(\mu_{j}, \eta_{j}, v_{j}\right)(j=1,2, \mathrm{~K}, n)$ be a collection of PFNs, and $P=\left(p_{1}, p_{2}, \ldots, p_{n}\right) \in R^{n}$ be a vector of parameters, then the aggregated value by PFMM is still a PFN, and $\operatorname{PFMM}^{p}\left(\alpha_{1}, \alpha_{2}, \mathrm{~K}, \alpha_{n}\right)=$

$$
\left(\left(1-\prod_{\vartheta \in S_{n}}\left(1-\prod_{j=1}^{n} \mu_{\vartheta(j)}^{p_{j}}\right)^{\frac{1}{n !}}\right)^{\frac{1}{\sum_{j=1}^{n} p_{j}}},\right.
$$




$$
\begin{aligned}
& 1-\left(1-\prod_{\vartheta \in S_{n}}\left(1-\prod_{j=1}^{n}\left(1-\eta_{\vartheta(j)}\right)^{p_{j}}\right)^{\frac{1}{n !}}\right)^{\frac{1}{\sum_{j=1}^{n} p_{j}}}, \\
& 1-\left(1-\prod_{\vartheta \in S_{n}}\left(1-\prod_{j=1}^{n}\left(1-v_{\vartheta(j)}\right)^{p_{j}}\right)^{\frac{1}{n !}}\right)^{\frac{1}{\sum_{j=1}^{n} p_{j}}} .
\end{aligned}
$$

Proof. According to the operations for PFNs, we get

$$
\alpha_{\vartheta(j)}^{p_{j}}=\left(\mu_{\vartheta(j)}^{p_{j}}, 1-\left(1-\eta_{\vartheta(j)}\right)^{p_{j}}, 1-\left(1-v_{\vartheta(j)}\right)^{p_{j}}\right),
$$

and,

$$
\begin{aligned}
& \prod_{j=1}^{n} \alpha_{\vartheta(j)}^{p_{j}}=\left(\prod_{j=1}^{n} \mu_{\vartheta(j)}^{p_{j}}, 1-\prod_{j=1}^{n}\left(1-\eta_{\vartheta(j)}\right)^{p_{j}},\right. \\
&\left.1-\prod_{j=1}^{n}\left(1-v_{\vartheta(j)}\right)^{p_{j}}\right) .
\end{aligned}
$$

Therefore,

$$
\begin{aligned}
\sum_{\vartheta \in S_{n}} \prod_{j=1}^{n} \alpha_{\vartheta(j)}^{p_{j}}=\left(1-\prod_{\vartheta \in S_{n}}\left(1-\prod_{j=1}^{n} \mu_{\vartheta(j)}^{p_{j}}\right),\right. \\
\prod_{\vartheta \in S_{n}}\left(1-\prod_{j=1}^{n}\left(1-\eta_{\vartheta(j)}\right)^{p_{j}}\right), \prod_{\vartheta \in S_{n}}\left(1-\prod_{j=1}^{n}\left(1-v_{\vartheta(j)}\right)^{p_{j}}\right),
\end{aligned}
$$

and,

$$
\begin{aligned}
& \frac{1}{n !} \sum_{\vartheta \in S_{n}} \prod_{j=1}^{n} \alpha_{\vartheta(j)}^{p_{j}}= \\
& \left(1-\prod_{\vartheta \in S_{n}}\left(1-\prod_{j=1}^{n} \mu_{\vartheta(j)}^{p_{j}}\right)^{\frac{1}{n !}},\right. \\
& \prod_{\vartheta \in S_{n}}\left(1-\prod_{j=1}^{n}\left(1-\eta_{\vartheta(j)}\right)^{p_{j}}\right)^{\frac{1}{n !}}, \\
& \left.\prod_{\vartheta \in S_{n}}\left(1-\prod_{j=1}^{n}\left(1-v_{\vartheta(j)}\right)^{p_{j}}\right)^{\frac{1}{n !}}\right) .
\end{aligned}
$$

Thus,

$$
\begin{aligned}
& \left(\frac{1}{n !} \sum_{\vartheta \in S_{n}} \prod_{j=1}^{n} \alpha_{\vartheta(j)}^{p_{j}}\right)^{\frac{1}{\sum_{j=1}^{n} p_{j}}}= \\
& \left(\left(1-\prod_{\vartheta \in S_{n}}\left(1-\prod_{j=1}^{n} \mu_{\vartheta(j)}^{p_{j}}\right)^{\frac{1}{n !}}\right)^{\frac{1}{\sum_{j=1}^{n} p_{j}}},\right.
\end{aligned}
$$

$$
\begin{aligned}
& 1-(1- \prod_{\vartheta \in S_{n}}\left(1-\prod_{j=1}^{n}\left(1-\eta_{\vartheta(j)}\right)^{p_{j}}\right)^{\left.\frac{1}{n !}\right)^{\frac{1}{\sum_{j=1}^{n} p_{j}}},} \\
&\left.1-\left(1-\prod_{\vartheta \in S_{n}}\left(1-\prod_{j=1}^{n}\left(1-v_{\vartheta(j)}\right)^{p_{j}}\right)^{\frac{1}{n !}}\right)^{\frac{1}{\sum_{j=1}^{n} p_{j}}}\right),
\end{aligned}
$$

which completes the proof of Theorem 1.

\section{Example 1.}

Let $\quad \alpha_{1}=(0.2,0.3,0.4) \quad, \quad \alpha_{1}=(0.1,0.4,0.3)$, and $\alpha_{3}=(0.3,0.5,0.1)$ be three PFNs, then if we utilize PFMM operator to aggregate these three PFNs, the calculation process is shown as follows. Here we assume $P=(1,2,3)$.

PFMM has the ability of capturing the interrelationships among all input PFSs. In addition, PFMM makes the information aggregation process flexible. By assigning different values to the vector of parameters, different cases can be derived. In the followings, we investigate some special cases of PFMM with respect of the vector of parameters $P$.

Case 1: if $P=(1,0, \ldots, 0)$, then the PFMM operator reduces to the following

$$
\begin{aligned}
\operatorname{PFMM}^{(1,0, \mathrm{~K}, 0)}\left(\alpha_{1}, \alpha_{2}, \mathrm{~K}, \alpha_{n}\right)=\frac{1}{n} \sum_{j=1}^{n} \alpha_{j}= \\
\left(1-\prod_{j=1}^{n}\left(1-\mu_{j}\right)^{1 / n}, \prod_{j=1}^{n} \eta_{j}^{1 / n}, \prod_{j=1}^{n} v_{j}^{1 / n}\right),
\end{aligned}
$$

which is the picture fuzzy arithmetic averaging (PFA) operator.

Case 2: if $P=(\lambda, 0, \ldots, 0)$, then the PFMM operator reduces to the following

$$
\begin{gathered}
\operatorname{PFMM}^{(\lambda, 0, \mathrm{~K}, 0)}\left(\alpha_{1}, \alpha_{2}, \mathrm{~K}, \alpha_{n}\right)=\left(\frac{1}{n} \sum_{j=1}^{n} \alpha_{j}^{\lambda}\right)^{1 / \lambda}= \\
\left(\left(1-\prod_{j=1}^{n}\left(1-\mu_{j}^{\lambda}\right)^{1 / n}\right)^{1 / \lambda}, 1-\left(1-\prod_{j=1}^{n}\left(1-\left(1-\eta_{j}\right)^{\lambda}\right)^{1 / n}\right)^{1 / \lambda},\right. \\
\left.1-\left(1-\prod_{j=1}^{n}\left(1-\left(1-v_{j}\right)^{\lambda}\right)^{1 / n}\right)^{1 / \lambda}\right),
\end{gathered}
$$

which is the generalized picture fuzzy arithmetic averaging (GPFA) operator.

Case 3: if $P=(1,1,0,0, \ldots, 0)$, then the PFMM operator reduces to the following 


$$
\begin{gathered}
\operatorname{PFMM}^{(1,1,0,0, \mathrm{~K}, 0)}\left(\alpha_{1}, \alpha_{2}, \mathrm{~K}, \alpha_{n}\right)=\left(\frac{1}{n(n-1)} \sum_{\substack{i, j=1 \\
i \neq j}}^{n} \alpha_{i} \alpha_{j}\right)^{\frac{1}{2}} \\
=\left(\left(1-\prod_{\substack{i, j \neq 1 \\
i \neq j}}^{n}\left(1-\mu_{i} \mu_{j}\right)^{\frac{1}{n(n-1)}}\right)^{\frac{1}{2}},\right. \\
1-\left(1-\prod_{\substack{i, j \neq 1 \\
i \neq j}}^{n}\left(\eta_{i}+\eta_{j}-\eta_{i} \eta_{j}\right)^{\frac{1}{n(n-1)}}\right)^{\frac{1}{2}}, \\
\left.1-\left(1-\prod_{\substack{i, j \neq 1 \\
i \neq j}}^{n}\left(v_{i}+v_{j}-v_{i} v_{j}\right)^{\frac{1}{n(n-1)}}\right)^{\frac{1}{2}}\right),(8)
\end{gathered}
$$

which is the picture fuzzy Bonferroni mean (PFBM) operator.

$$
647 k \quad 8 \quad 647-k 48
$$

Case 4: if $P=(1,1, \ldots, 1,0,0, \ldots, 0)$, then the PFMM operator reduces to the following

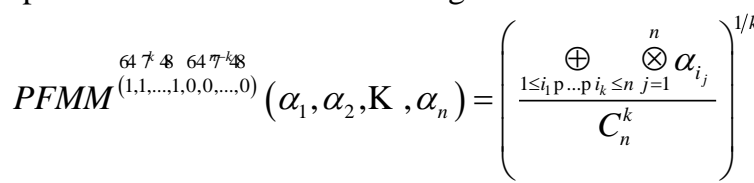

$$
\begin{aligned}
& =\left(\left(1-\prod_{1 \leq i_{\mathrm{p}} \mathrm{pK} i_{k} \leq n}\left(1-\prod_{j=1}^{n} \mu_{i_{j}}\right)^{\frac{1}{c_{n}^{k}}}\right)^{1 / k},\right. \\
& 1-\left(1-\prod_{1 \leq i_{\mathrm{p}} \mathrm{p} \mathrm{p}_{k} \leq n}\left(1-\prod_{j=1}^{n}\left(1-\eta_{i_{j}}\right)\right)^{\frac{1}{c_{n}^{k}}}\right)^{1 / k}, \\
& \left.1-\left(1-\prod_{1 \leq i_{1} \mathrm{pK} \mathrm{p} i_{k} \leq n}\left(1-\prod_{j=1}^{n}\left(1-v_{i_{j}}\right)\right)^{\frac{1}{c_{n}^{k}}}\right)^{1 / k}\right),
\end{aligned}
$$

which is the picture fuzzy Maclaurin symmetric mean (PFMSM) operator.

Case 5: if $P=(1,1, \ldots, 1)$, then the PFMM operator reduces to the following

$$
\begin{aligned}
\operatorname{PFMM}^{(1,1, \ldots, 1)}\left(\alpha_{1}, \alpha_{2}, \mathrm{~K}, \alpha_{n}\right)=\prod_{j=1}^{n} \alpha_{j}^{1 / n} \\
=\left(\prod_{j=1}^{n} u_{j}^{1 / n}, 1-\prod_{j=1}^{n}\left(1-\eta_{j}\right)^{1 / n}, 1-\prod_{j=1}^{n}\left(1-v_{j}\right)^{1 / n}\right),
\end{aligned}
$$

which is the picture fuzzy geometric averaging (PFG) operator.

Case 6: if $P=(1 / n, 1 / n, \ldots, 1 / n)$, then the PFMM operator reduces to the following

$$
\begin{aligned}
P^{\prime} & M^{(1 / n, 1 / n, \ldots, 1 / n)}\left(\alpha_{1}, \alpha_{2}, \mathrm{~K}, \alpha_{n}\right)=\prod_{j=1}^{n} \alpha_{j}^{1 / n} \\
= & \left(\prod_{j=1}^{n} u_{j}^{1 / n}, 1-\prod_{j=1}^{n}\left(1-\eta_{j}\right)^{1 / n}, 1-\prod_{j=1}^{n}\left(1-v_{j}\right)^{1 / n}\right),
\end{aligned}
$$

which is the picture fuzzy geometric averaging (PFG) operator.

In the followings, we discuss some desirable properties of the PFMM operator.

Theorem 2. (Idempotency) Let $\alpha_{j}=\left(\mu_{j}, \eta_{j}, v_{j}\right)$, $j=1,2, \mathrm{~K}, n$ be a collection of PFNs, if all the PFNs are equal, i.e. $\alpha_{j}=\alpha=(\mu, \eta, v)$, then

$$
\operatorname{PFMM}^{p}\left(\alpha_{1}, \alpha_{2}, \mathrm{~K}, \alpha_{n}\right)=\alpha \text {. }
$$

Proof. Since $\alpha_{j}=\alpha=(u, \eta, v)$, according to Theorem 1 , we have

$$
\begin{aligned}
& \operatorname{PFMM}^{p}\left(\alpha_{1}, \alpha_{2}, \mathrm{~K}, \alpha_{n}\right) \\
& =\left(\left(1-\prod_{\vartheta \in S_{n}}\left(1-\prod_{j=1}^{n} \mu^{p_{j}}\right)^{\frac{1}{n !}}\right)^{\frac{1}{\sum_{j=1}^{n} p_{j}}},\right. \\
& 1-\left(1-\prod_{\vartheta \in S_{n}}\left(1-\prod_{j=1}^{n}(1-\eta)^{p_{j}}\right)^{\frac{1}{n !}}\right)^{\frac{1}{n} p_{j=1}}, \\
& 1-\left(1-\prod_{g \in S_{n}}\left(1-\prod_{j=1}^{n}(1-v)^{p_{j}}\right)^{\frac{1}{n !}} \sum_{j=1}^{\frac{1}{n} p_{j}}\right) \\
& =\left(\left(1-\prod_{\vartheta \in S_{n}}\left(1-\mu^{\sum_{j=1}^{n} p_{j}}\right)^{\frac{1}{n !}}\right)^{\frac{1}{\sum_{j=1}^{n} p_{j}}},\right. \\
& 1-\left(1-\prod_{\vartheta \in S_{n}}\left(1-(1-\eta)_{j=1}^{n} p_{j}\right)^{\left.\frac{1}{n !}\right)^{\frac{1}{\sum_{j=1}^{n} p_{j}}},}\right.
\end{aligned}
$$




$$
\begin{aligned}
& \left.1-\left(1-\prod_{\vartheta \in S_{n}}\left(1-(1-v) \sum_{j=1}^{n} p_{j}\right)^{\frac{1}{n !}}\right)_{j=1}^{\frac{1}{n} p_{j}}\right) \\
& =\left(\left(1-\left(\left(1-\mu^{\sum_{j=1}^{n} p_{j}}\right)^{n !}\right)^{\frac{1}{n !}}\right)^{\frac{1}{n} \sum_{j=1}^{n} p_{j}},\right. \\
& 1-\left(1-\left(\left(1-(1-\eta) \sum_{j=1}^{n} p_{j}\right)^{n !}\right)\right)^{\frac{1}{n !}}, \\
& \left.1-\left(1-\left(\left(1-(1-v) \sum_{j=1}^{n} p_{j}\right)^{n !}\right)^{\frac{1}{n !}}\right)^{\frac{1}{\sum_{j=1}^{n} p_{j}}}\right) \\
& =\left(\left(1-\left(1-\mu^{\sum_{j=1}^{n} p_{j}}\right)\right)^{\frac{1}{n} p_{j=1}^{n}}, 1-\left((1-\eta) \sum_{j=1}^{n} p_{j}\right) \sum_{j=1}^{\frac{1}{n} p_{j}},\right. \\
& \left.1-\left((1-v) \sum_{j=1}^{n} p_{j}\right) \frac{1}{\sum_{j=1}^{n} p_{j}}\right) \\
& =\left(\left(\mu^{\sum_{j=1}^{n} p_{j}}\right)_{j=1}^{\frac{1}{n} \sum_{j}^{n} p_{j}}, 1-(1-\eta), 1-(1-v)\right) \\
& =(\mu, \eta, v) \text {. }
\end{aligned}
$$

Theorem 3. (Monotonicity) Let $\alpha_{j}=\left(\mu_{j}, \eta_{j}, v_{j}\right)$ and $\alpha_{j}^{\prime}=\left(\mu_{j}^{\prime}, \eta_{j}^{\prime}, v_{j}^{\prime}\right)$ be two sets of PFNs, If $\mu_{j} \geq \mu_{j}^{\prime}, \eta_{j} \geq \eta_{j}^{\prime}, v_{j} \geq v_{j}^{\prime}$ for all $j$, then $\operatorname{PFMM}^{P}\left(\alpha_{1}, \alpha_{2}, \mathrm{~K}, \alpha_{n}\right) \geq \operatorname{PFMM}^{P}\left(\alpha_{1}^{\prime}, \alpha_{2}^{\prime}, \mathrm{K}, \alpha_{n}^{\prime}\right)$,

Proof. Let $\operatorname{PFMM}^{P}\left(\alpha_{1}, \alpha_{2}, \mathrm{~K}, \alpha_{n}\right)=(\mu, \eta, v)$, $\operatorname{PFMM}^{P}\left(\alpha_{1}^{\prime}, \alpha_{2}^{\prime}, \mathrm{K}, \alpha_{n}^{\prime}\right)=\left(\mu^{\prime}, \eta^{\prime}, v^{\prime}\right)$, where

$$
\mu=\left(1-\prod_{\vartheta \in S_{n}}\left(1-\prod_{j=1}^{n} \mu_{\vartheta(j)}^{p_{j}}\right)^{\frac{1}{n !}}\right)^{\frac{1}{n_{j=1}^{n}} p_{j}},
$$

$$
\mu^{\prime}=\left(1-\prod_{\vartheta \in S_{n}}\left(1-\prod_{j=1}^{n} \mu_{\vartheta(j)}^{\prime p_{j}}\right)^{\frac{1}{n !}}\right)^{\frac{1}{n_{j=1}^{n} p_{j}}},
$$

and

$$
\begin{aligned}
& \eta=1-\left(1-\prod_{\vartheta \in S_{n}}\left(1-\prod_{j=1}^{n}\left(1-\eta_{\vartheta(j)}\right)^{p_{j}}\right)^{\frac{1}{n !}}\right)^{\frac{1}{n} \sum_{j=1}^{n} p_{j}}, \\
& \eta^{\prime}=1-\left(1-\prod_{\vartheta \in S_{n}}\left(1-\prod_{j=1}^{n}\left(1-\eta_{\vartheta(j)}^{\prime}\right)^{p_{j}}\right)^{\frac{1}{n !}}\right)^{\sum_{j=1}^{\frac{1}{n} p_{j}}},
\end{aligned}
$$

and

$$
\begin{aligned}
& v=1-\left(1-\prod_{\vartheta \in S_{n}}\left(1-\prod_{j=1}^{n}\left(1-v_{\vartheta(j)}\right)^{p_{j}}\right)^{\frac{1}{n !}}\right)^{\frac{1}{n} \sum_{j=1}^{n} p_{j}}, \\
& v^{\prime}=1-\left(1-\prod_{\vartheta \in S_{n}}\left(1-\prod_{j=1}^{n}\left(1-v_{\vartheta(j)}^{\prime}\right)^{p_{j}}\right)^{\frac{1}{n !}}\right)^{\frac{1}{n} p^{\frac{1}{n}} p_{j}} .
\end{aligned}
$$

Since $\mu_{j} \geq \mu_{j}^{\prime}$, we can get

$$
\begin{gathered}
\mu_{\vartheta(j)}^{p_{j}} \geq \mu_{\vartheta(j)}^{\prime p_{j}} \text { and } \prod_{j=1}^{n} \mu_{\vartheta(j)}^{p_{p_{j}}} \geq \prod_{j=1}^{n} \mu_{\vartheta(j)}^{\prime p_{j}}, \\
1-\prod_{j=1}^{n} \mu_{\vartheta(j)}^{p_{j}} \leq 1-\prod_{j=1}^{n} \mu_{\vartheta(j)}^{p_{p_{j}}}, \\
\prod_{\vartheta \in S_{n}}\left(1-\prod_{j=1}^{n} \mu_{\vartheta(j)}^{p_{j}}\right) \leq \prod_{\vartheta \in S_{n}}\left(1-\prod_{j=1}^{n} \mu_{\vartheta(j)}^{p_{j_{j}}}\right) .
\end{gathered}
$$

Then,

$$
\begin{gathered}
\prod_{\vartheta \in S_{n}}\left(1-\prod_{j=1}^{n} \mu_{\vartheta(j)}^{p_{j}}\right)^{\frac{1}{n !}} \leq \prod_{\vartheta \in S_{n}}\left(1-\prod_{j=1}^{n} \mu_{\vartheta(j)}^{\prime p_{j}}\right)^{\frac{1}{n !}}, \\
1-\prod_{\vartheta \in S_{n}}\left(1-\prod_{j=1}^{n} \mu_{\vartheta(j)}^{p_{j}}\right)^{\frac{1}{n !}} \geq 1-\prod_{\vartheta \in S_{n}}\left(1-\prod_{j=1}^{n} \mu_{\vartheta(j)}^{\prime p_{j}}\right)^{\frac{1}{n !}} .
\end{gathered}
$$

Further,

$\left(1-\prod_{\vartheta \in S_{n}}\left(1-\prod_{j=1}^{n} \mu_{\vartheta(j)}^{p_{j}}\right)^{\frac{1}{n !}}\right)^{\frac{1}{\sum_{j=1}^{n} p_{j}}} \geq$

which means $\mu \geq \mu^{\prime}$.

$$
\left(1-\prod_{\vartheta \in S_{n}}\left(1-\prod_{j=1}^{n} \mu_{\vartheta(j)}^{\prime_{p_{j}}}\right)^{\frac{1}{n !}}\right)^{\frac{1}{\sum_{j=1}^{n} p_{j}}},
$$


Similarly, we can get $\eta \geq \eta^{\prime}, v \geq v^{\prime}$. Thus, according to the comparison law for PFNs, we can obtain

$\operatorname{PFMM}^{P}\left(\alpha_{1}, \alpha_{2}, \mathrm{~K}, \alpha_{n}\right) \geq \operatorname{PFMM}^{P}\left(\alpha_{1}^{\prime}, \alpha_{2}^{\prime}, \mathrm{K}, \alpha_{n}^{\prime}\right)$.

Theorem 4. (Boundedness) Let $\alpha_{j}=\left(\mu_{j}, \eta_{j}, v_{j}\right)$, $j=1,2, \mathrm{~K}, n$ be a collection of PFNs, if $\alpha^{+}=\max _{j} \alpha_{j}$ and $\alpha^{-}=\min _{j} \alpha_{j}$, then

$$
\alpha^{-} \leq \operatorname{PFMM}^{P}\left(\alpha_{1}, \alpha_{2}, \ldots, \alpha_{n}\right) \leq \alpha^{+} .
$$

Proof. According to Theorem 2 and Theorem 3, we can get

$\operatorname{PFMM}^{P}\left(\alpha_{1}, \alpha_{2}, \ldots, \alpha_{n}\right) \geq \operatorname{PFMM}^{P}\left(\alpha^{-}, \alpha^{-}, \ldots, \alpha^{-}\right)=\alpha^{-}$ and

$$
\operatorname{PFMM}^{P}\left(\alpha_{1}, \alpha_{2}, \ldots, \alpha_{n}\right) \geq \operatorname{PFMM}^{P}\left(\alpha^{+}, \alpha^{+}, \ldots, \alpha^{+}\right)=\alpha^{+}
$$

Thus, we can get

$$
\alpha^{-} \leq \operatorname{PFMM}^{P}\left(\alpha_{1}, \alpha_{2}, \ldots, \alpha_{n}\right) \leq \alpha^{+} .
$$

\subsection{The picture fuzzy weighted Muirhead mean operator}

The advantage of the PFMM is that it can consider the interrelationship between the aggregated PFNs. However, PFMM does not consider the selfimportance of the aggregated arguments. Therefore, we introduce picture fuzzy weighted Muirhead mean (PFWMM) operator which can take the corresponding weights of aggregated PFNs into consideration.

Definition 7. Let $\alpha_{j}=\left(\mu_{j}, \eta_{j}, v_{j}\right)(j=1,2, \ldots, n)$ be a collection of PFNs, and $P=\left(p_{1}, p_{2}, \ldots, p_{n}\right) \in R^{n}$ be a vector of parameters, Let $w=\left(w_{1}, w_{2}, \ldots, w_{n}\right)^{T}$ be the weight vector of $\alpha_{j}(j=1,2, \ldots, n)$, satisfying $0 \leq w_{j} \leq 1$ and $\sum_{j=1}^{n} w_{j}=1$. Then PFWMM operator is defined as

$$
\begin{aligned}
\operatorname{PFWMM}^{P}\left(\alpha_{1}, \alpha_{2}, \ldots, \alpha_{n}\right)= \\
\qquad\left(\frac{1}{n !} \sum_{\vartheta \in S_{n}} \prod_{j=1}^{n}\left(n w_{\vartheta(j)} \alpha_{\vartheta(j)}\right)^{p_{j}}\right)^{\frac{1}{\sum_{j=1}^{n} p_{j}}},
\end{aligned}
$$

then $P F W M M^{P}$ is called the PFWMM, where $\vartheta(j)(j=1,2, \ldots, n)$ is any permutation of $(1,2, \mathrm{~K}, n)$, and $S_{n}$ is the collection of all permutation of $(1,2, \mathrm{~K}, n)$.
According to the operations of PFNs, the aggregated value by the PFWMM can be obtained, which is shown as Theorem 5.

Theorem 5. Let $\alpha_{j}=\left(\mu_{j}, \eta_{j}, v_{j}\right)(j=1,2, \ldots, n)$ be a collection of PFNs and $P=\left(p_{1}, p_{2}, \ldots, p_{n}\right) \in R^{n}$ be a vector of parameters, then the aggregated value by the PFWMM is also a PFN and

$\operatorname{PFWMM}^{P}\left(\alpha_{1}, \alpha_{2}, \ldots, \alpha_{n}\right)=$

$$
\begin{aligned}
\left(\left(1-\left(\prod_{\vartheta \in S_{n}}\left(1-\prod_{j=1}^{n}\left(1-\left(1-\mu_{\vartheta(j)}\right)^{n w_{\vartheta(j)}}\right)^{p_{j}}\right)\right)^{\frac{1}{n !}}\right)^{\frac{1}{\sum_{j=1}^{n} P_{j}}},\right. \\
1-\left(1-\left(\prod_{\vartheta \in S_{n}}\left(1-\prod_{j=1}^{n}\left(1-\eta_{\vartheta(j)}^{n w_{\vartheta(j)}}\right)^{p_{j}}\right)\right)^{\frac{1}{n !}}\right)^{\frac{1}{\sum_{j=1}^{n} P_{j}}}, \\
1-\left(1-\left(\prod_{\vartheta \in S_{n}}\left(1-\prod_{j=1}^{n}\left(1-v_{\vartheta(j)}^{n w_{\vartheta(j)}}\right)^{p_{j}}\right)\right)^{\frac{1}{n !}}\right)^{\frac{1}{\sum_{j=1}^{n} P_{j}}} .
\end{aligned}
$$

Proof. Because $n w_{\vartheta(j)} \alpha_{\vartheta(j)}=\left(1-\left(1-\mu_{\vartheta(j)}\right)^{n w_{\vartheta(j)}}\right.$, $\left.\eta_{\vartheta(j)}^{n w_{\vartheta(j)}}, v_{\vartheta(j)}^{n w_{\vartheta(j)}}\right)$, we can replace $\mu_{\vartheta(j)}$ in Eq. (5) with $1-\left(1-\mu_{\vartheta(j)}\right)^{n w_{\vartheta(j)}}$, and $\eta_{\vartheta(j)}$ in Eq. (5) with $\eta_{\vartheta(j)}^{n w_{\vartheta(j)}}$, and $v_{\vartheta(j)}$ in Eq. (5) with $v_{\vartheta(j)}^{n w_{\vartheta(j)}}$, then we can get Eq. (16).

Because $\alpha_{\vartheta(j)}$ is a PFN, $n w_{\vartheta(j)} \alpha_{\vartheta(j)}$ is also a PFN. By Eq. (5), we know $\operatorname{PFWMM}^{P}\left(\alpha_{1}, \alpha_{2}, \ldots, \alpha_{n}\right)$ is a PFN.

Flowingly, we present some desirable properties of the PFWMM operator.

Theorem 6. (Monotonicity) Let $\alpha_{j}=\left(\mu_{j}, \eta_{j}, v_{j}\right)$ and $\alpha_{j}^{\prime}=\left(\mu_{j}^{\prime}, \eta_{j}^{\prime}, v_{j}^{\prime}\right)(j=1,2, \mathrm{~K}, n)$ be two sets of PFNs. If $\alpha_{j} \geq \alpha_{j}^{\prime}$ for all $j$, then

$\operatorname{PFWMM}^{P}\left(\alpha_{1}, \alpha_{2}, \ldots, \alpha_{n}\right) \geq \operatorname{PFWMM}^{P}\left(\alpha_{1}^{\prime}, \alpha_{2}^{\prime}, \ldots, \alpha_{n}^{\prime}\right)$

The proof of Theorem 6 is similar to that of Theorem 3 therefore we will skip it here to avoid repetition.. 
Theorem 7. (Boundedness) Let $\alpha_{j}=\left(\mu_{j}, \eta_{j}, v_{j}\right)$, $(j=1,2, \ldots, n)$ be a collection of PFNs, if $\alpha^{+}=\max _{j} \alpha_{j}$ and $\alpha^{-}=\min _{j} \alpha_{j}$, then

$$
\alpha^{-} \leq P F W M M^{P}\left(\alpha_{1}, \alpha_{2}, \ldots, \alpha_{n}\right) \leq \alpha^{+},
$$

Evidently, the PFWMM operator does not has the property of idempotency.

\subsection{The picture fuzzy dual Muirhead mean operator}

In this section, we extend the DMM to aggregate picture fuzzy information.

Definition 8. Let $\alpha_{j}=\left(\mu_{j}, \eta_{j}, v_{j}\right)(j=1,2, \ldots, n)$ be a collection of PFNs, and $P=\left(p_{1}, p_{2}, \ldots, p_{n}\right) \in R^{n}$ be a vector of parameters, then the PFDMM is defined as $\operatorname{PFDMM}^{P}\left(\alpha_{1}, \alpha_{2}, \ldots, \alpha_{n}\right)$

$$
=\frac{1}{\sum_{j=1}^{n} p_{j}}\left(\prod_{\vartheta \in S_{n}} \sum_{j=1}^{n}\left(p_{j} \alpha_{\vartheta(j)}\right)\right)^{\frac{1}{n !}},
$$

where $\vartheta(j)(j=1,2, \ldots, n)$ is any permutation of $(1,2, \mathrm{~K}, n)$, and $S_{n}$ is the collection of all permutation of $(1,2, \mathrm{~K}, n)$.

Theorem 8. Let $\alpha_{j}=\left(\mu_{j}, \eta_{j}, v_{j}\right)(j=1,2, \ldots, n)$ be a collection of PFNs and $P=\left(p_{1}, p_{2}, \ldots, p_{n}\right) \in R^{n}$ be a vector of parameters, then the aggregated value by the PFDMM is also a PFN and

$\operatorname{PFDMM}^{P}\left(\alpha_{1}, \alpha_{2}, \ldots, \alpha_{n}\right)=$

$$
\begin{aligned}
&\left(1-\left(1-\prod_{\vartheta \in S_{n}}\left(1-\prod_{j=1}^{n}\left(1-\mu_{\vartheta(j)}\right)^{p_{j}}\right)^{\frac{1}{n !}}\right)^{\frac{1}{\sum_{j=1}^{n} p_{j}}},\right. \\
&\left(1-\prod_{\vartheta \in S_{n}}\left(1-\prod_{j=1}^{n} \eta_{\vartheta(j)}^{p_{j}}\right)^{\left.\frac{1}{n !}\right)^{\frac{1}{\sum_{j=1}^{n} p_{j}}},}\right. \\
&\left.\left(1-\prod_{\vartheta \in S_{n}}\left(1-\prod_{j=1}^{n} v_{\vartheta(j)}^{p_{j}}\right)^{\frac{1}{n !}}\right)^{\frac{1}{\sum_{j=1}^{n} p_{j}}}\right),
\end{aligned}
$$

The proof of Theorem 8 is similar to that of Theorem 1 , which is skipped here to save space.
In the followings, we will discuss some special cases of PFDMM regarding of the parameter vector $P$.

Case 1: if $P=(1,0, \ldots, 0)$, then the PFDMM operator reduces to the following

$$
\begin{aligned}
& \operatorname{PFDMM}^{(1,0, \ldots, 0)}\left(\alpha_{1}, \alpha_{2}, \ldots, \alpha_{n}\right)=\prod_{j=1}^{n} \alpha_{j}^{1 / n}= \\
& \left(\prod_{j=1}^{n} u_{j}^{1 / n}, 1-\prod_{j=1}^{n}\left(1-\eta_{j}\right)^{1 / n}, 1-\prod_{j=1}^{n}\left(1-v_{j}\right)^{1 / n}\right),
\end{aligned}
$$

which is the PFG operator.

Case 2: if $P=(\lambda, 0, \ldots, 0)$, then the PFDMM operator reduces to the following

$$
\begin{gathered}
\operatorname{PFDMM}^{(\lambda, 0, \ldots, 0)}\left(\alpha_{1}, \alpha_{2}, \ldots, \alpha_{n}\right)=\frac{1}{\lambda}\left(\prod_{j=1}^{n}\left(\lambda \alpha_{j}\right)^{\frac{1}{n}}\right)= \\
\left(1-\left(1-\prod_{j=1}^{n}\left(1-\left(1-\mu_{j}\right)^{\lambda}\right)^{1 / n}\right)^{1 / \lambda},\left(1-\prod_{j=1}^{n}\left(1-\eta_{j}^{\lambda}\right)^{1 / n}\right)^{1 / \lambda},\right. \\
\left.\left(1-\prod_{j=1}^{n}\left(1-v_{j}^{\lambda}\right)^{1 / n}\right)^{1 / \lambda}\right),
\end{gathered}
$$

which is the generalized picture fuzzy geometric (GPFG) operator.

Case 3: if $P=(1,1,0,0, \ldots, 0)$, then the PFDMM operator reduces to the following

$$
\begin{aligned}
& \operatorname{PFDMM}^{(1,1,0,0, \mathrm{~K}, 0)}\left(\alpha_{1}, \alpha_{2}, \mathrm{~K}, \alpha_{n}\right)=\frac{1}{2} \prod_{\substack{i, j=1 \\
i \neq j}}^{n}\left(\alpha_{i} \oplus \alpha_{j}\right)^{\frac{1}{n(n-1)}} \\
& =\left(1-\left(1-\prod_{\substack{i, j \neq 1 \\
i \neq j}}^{n}\left(\mu_{i}+\mu_{j}-\mu_{i} \mu_{j}\right)^{\frac{1}{n(n-1)}}\right)^{\frac{1}{2}},\right. \\
& \left(\begin{array}{l}
\left.1-\prod_{\substack{i, j \neq 1 \\
i \neq j}}^{n}\left(1-\eta_{i} \eta_{j}\right)^{\frac{1}{n(n-1)}}\right)^{\frac{1}{2}}, \\
\left.\left.1-\prod_{\substack{i, j \neq 1 \\
i \neq j}}^{n}\left(1-v_{i} v_{j}\right)^{\frac{1}{n(n-1)}}\right)^{\frac{1}{2}}\right),(22)
\end{array}\right)
\end{aligned}
$$

which is the picture fuzzy geometric Bonferroni mean (PFGBM) operator.

$$
647^{k} \quad 8 \quad 64 \pi-k 48
$$

Case 4: if $P=(1,1, \ldots, 1,0,0, \ldots, 0)$, then the PFDMM operator reduces to the following 


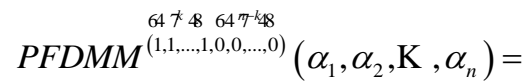

$$
\begin{aligned}
& \left(1-\left(1-\prod_{1 \leq i_{1} \mathrm{pK} \mathrm{p} i_{k} \leq n}\left(1-\prod_{j=1}^{n}\left(1-\mu_{i_{j}}\right)\right)^{\frac{1}{C_{n}^{k}}}\right)^{1 / k},\right. \\
& \left(1-\prod_{1 \leq i_{1} \mathrm{pK} \mathrm{p} i_{k} \leq n}\left(1-\prod_{j=1}^{n} \eta_{i_{j}}\right)^{\frac{1}{C_{n}^{k}}}\right)^{1 / k} \\
& \left.\left(1-\prod_{1 \leq i_{1} \mathrm{pK} i_{i_{k}} \leq n}\left(1-\prod_{j=1}^{n} v_{i_{j}}\right)^{\frac{1}{C_{n}^{k}}}\right)^{1 / k}\right) \\
& =\frac{1}{k}\left(\underset{1 \leq i_{1} \mathrm{p} \ldots \mathrm{p} i_{k} \leq k}{\otimes}\left(\bigoplus_{j=1}^{k} \alpha_{i_{j}}\right)^{\frac{1}{C_{n}^{k}}}\right),
\end{aligned}
$$

which is the picture fuzzy dual Maclaurin symmetric mean (PFDMSM) operator.

Case 5: if $P=(1,1, \ldots, 1)$, then the PFDMM operator reduces to the following

$$
\begin{aligned}
\operatorname{PFDMM}^{(1,1, \ldots, 1)}\left(\alpha_{1}, \alpha_{2}, \mathrm{~K}, \alpha_{n}\right)=\frac{1}{n} \sum_{j=1}^{n} \alpha_{j}= \\
\left(1-\prod_{j=1}^{n}\left(1-\mu_{j}\right)^{1 / n}, \prod_{j=1}^{n} \eta_{j}^{1 / n}, \prod_{j=1}^{n} v_{j}^{1 / n}\right),
\end{aligned}
$$

which is the PFA operator.

Case 6: if $P=(1 / n, 1 / n, \ldots, 1 / n)$, then the PFDMM operator reduces to the following

$$
\begin{array}{r}
\operatorname{PFDMM}^{(1 / n, 1 / n, \ldots, 1 / n)}\left(\alpha_{1}, \alpha_{2}, \mathrm{~K}, \alpha_{n}\right)=\frac{1}{n} \sum_{j=1}^{n} \alpha_{j}= \\
\left(1-\prod_{j=1}^{n}\left(1-\mu_{j}\right)^{1 / n}, \prod_{j=1}^{n} \eta_{j}^{1 / n}, \prod_{j=1}^{n} v_{j}^{1 / n}\right),
\end{array}
$$

which is the PFA operator.

\subsection{The picture fuzzy weighted dual Muirhead mean operator}

Definition 9. Let $\alpha_{j}=\left(\mu_{j}, \eta_{j}, v_{j}\right)(j=1,2, \ldots, n)$ be a collection of PFNs, $w=\left(w_{1}, w_{2}, \ldots, w_{n}\right)^{T}$ be the weight vector of $\alpha_{j}(j=1,2, \ldots, n)$, satisfying $w_{i} \in[0,1]$ and $\sum_{j=1}^{n} w_{j}=1$, and let $P=\left(p_{1}, p_{2}, \ldots, p_{n}\right) \in R^{n}$ be a vector of parameters. If

$$
\operatorname{PFWDMM}^{P}\left(\alpha_{1}, \alpha_{2}, \ldots, \alpha_{n}\right)=
$$

$$
\frac{1}{\sum_{j=1}^{n} p_{j}}\left(\prod_{\vartheta \in S_{n}} \sum_{j=1}^{n}\left(p_{j} \alpha_{\vartheta(j)}^{n w_{\vartheta(j)}}\right)\right)^{\frac{1}{n !}},
$$

then $P F W D M M^{P}$ is the called the (picture fuzzy weighted dual Muirhead mean) PFWDMM operator, where $\vartheta(j)(j=1,2, \ldots, n)$ is any permutation of $(1,2, \mathrm{~K}, n)$, and $S_{n}$ is the collection of all permutation of $(1,2, \mathrm{~K}, n)$.

Theorem 9. Let $\alpha_{j}=\left(\mu_{j}, \eta_{j}, v_{j}\right)(j=1,2, \ldots, n)$ be a collection of PFNs and $P=\left(p_{1}, p_{2}, \ldots, p_{n}\right) \in R^{n}$ be a vector of parameters, then the aggregated value by using the PFWDMM operator is still a PFN and

$\operatorname{PFWDMM}^{P}\left(\alpha_{1}, \alpha_{2}, \ldots, \alpha_{n}\right)=$

$$
\begin{aligned}
& \left(1-\left(1-\prod_{\vartheta \in S_{n}}\left(1-\prod_{j=1}^{n}\left(1-\mu_{\vartheta(j)}^{n w_{\vartheta(j)}}\right)^{p_{j}}\right)^{\left.\frac{1}{n !}\right)^{\frac{1}{\sum_{j=1}^{n} p_{j}}},}\right.\right. \\
& \left(1-\prod_{\vartheta \in S_{n}}\left(1-\prod_{j=1}^{n}\left(1-\left(1-\eta_{\vartheta(j)}\right)^{n w_{\vartheta(j)}}\right)^{p_{j}}\right)^{\left.\frac{1}{n !}\right)^{\frac{1}{\sum_{j=1}^{n} p_{j}}},}\right. \\
& \left(1-\prod_{\vartheta \in S_{n}}\left(1-\prod_{j=1}^{n}\left(1-\left(1-v_{\vartheta(j)}\right)^{n w_{\vartheta(j)}}\right)^{p_{j}}\right)^{\frac{1}{n !}}\right)^{\frac{1}{\sum_{j=1}^{n} p_{j}}} .
\end{aligned}
$$

The proof is similar to that of Theorem 5, which is skipped to save space here.

In the following, we present some properties of the PFWDMM operator.

Theorem 10. (Monotonicity) Let $\alpha_{j}=\left(\mu_{j}, \eta_{j}, v_{j}\right)$ and $\alpha_{j}^{\prime}=\left(\mu_{j}^{\prime}, \eta_{j}^{\prime}, v_{j}^{\prime}\right)(j=1,2, \mathrm{~K}, n)$ be two sets of PFNs. If $\alpha_{j} \geq \alpha_{j}^{\prime}$ for all $\mathrm{j}$, then

$$
\begin{aligned}
\operatorname{PFWDMM}^{P}\left(\alpha_{1}, \alpha_{2}, \ldots, \alpha_{n}\right) & \\
& \geq \operatorname{PFWDMM}^{P}\left(\alpha_{1}^{\prime}, \alpha_{2}^{\prime}, \ldots, \alpha_{n}^{\prime}\right) .
\end{aligned}
$$

Theorem 11. (Boundedness) Let $\alpha_{j}=\left(\mu_{j}, \eta_{j}, v_{j}\right)$, $(j=1,2, \ldots, n)$ be a collection of PFNs, if $\alpha^{+}=\max _{j} \alpha_{j}$ and $\alpha^{-}=\min _{j} \alpha_{j}$, then

$$
\alpha^{-} \leq \operatorname{PFWMM}^{P}\left(\alpha_{1}, \alpha_{2}, \ldots, \alpha_{n}\right) \leq \alpha^{+} .
$$

Evidently, the PFWDMM operator does not has the property of idempotency. 


\section{An approach to MADM with picture fuzzy information}

In this section, we present a novel method to MADM in which attribute values take the form of PFNs. Consider a typical MADM problem in which $X=\left\{x_{1}, x_{2}, \ldots, x_{m}\right\}$ be a series of alternatives and $G=\left\{G_{1}, G_{2}, \ldots, G_{n}\right\}$ be a set of attributes, with the weight vector being $w=\left(w_{1}, w_{2}, \ldots, w_{n}\right)^{T}$, satisfying $\sum_{i=1}^{n} w_{i}=1$ and $w_{i} \in[0,1]$. For the attribute $G_{j}(j=1,2, \ldots, n)$ of alternative $x_{i}(i=1,2, \ldots, m)$, decision makers are required to use a PFN $p_{i j}=\left(\mu_{i j}, \eta_{i j}, v_{i j}\right) \quad i=1,2, \ldots, m ; j=1,2, \ldots, n$ to express their preference information. Therefore, a picture fuzzy decision matrix can be obtained. In the following section, we shall utilize the proposed picture fuzzy aggregation operators to solve this problem.

Step 1. Standardize the original decision matrix. Generally, attributes can be divided into two types, benefit attribute and cost attribute. Thus, the original decision matrix should be standardized as follows

$$
p_{i j}= \begin{cases}\left(\mu_{i j}, \eta_{i j}, v_{i j}\right) & G_{j} \in I_{1} \\ \left(v_{i j}, \eta_{i j}, \mu_{i j}\right) & G_{j} \in I_{2}\end{cases}
$$

where $I_{1}$ and $I_{2}$ represent the benefit type attribute and the cost type attribute respectively.

Step 2. For alternative $x_{i}(i=1,2, \mathrm{~K}, m)$, utilize the
Step 3. According to Definition 3, calculate the scores and accuracy of the overall preference value $p_{i}(i=1,2, \mathrm{~K}, m)$.

Step 4. Rank the alternatives.

\section{Numerical example}

In the following, we provide a numerical example to illustrate the application of the developed approach Suppose an organization plans to implement enterprise resource planning (ERP) system (adapted from [32]). The first step is to form a project team that consists of CIO (chief information officer) and two senior representatives from user departments. By collecting all possible information about ERP vendors and systems, project team chooses five potential ERP systems $x_{i}(i=1,2, \mathrm{~K}, 5)$ as candidates. The company employs some external professional organizations (or experts) to aid this decision-making. The project team selects four attributes to evaluate the alternatives: (1) function and technology $G_{1} ;$ (2) strategic fitness $G_{2}$; (3) vendor's ability $G_{3}$; (4) vendor's reputation $G_{4}$. The five possible ERP systems $X_{i}(i=1,2, \mathrm{~K}, 5)$ are to be evaluated using the picture fuzzy numbers by the decision makers under the above four attributes with the weighted vector $w=(0.2,0.1,0.3,0.4)^{T}$, and construct the following matrix $\alpha_{i j}=\left(\mu_{i j}, \eta_{i j}, v_{i j}\right)_{5 \times 4}$ shown in Table 1 .

Table 1. The picture fuzzy decision matrix

\begin{tabular}{lllll}
\hline & $G_{1}$ & $G_{2}$ & $G_{3}$ & $G_{4}$ \\
\hline$x_{1}$ & $(0.53,0.33,0.09)$ & $(0.89,0.08,0.03)$ & $(0.42,0.35,0.18)$ & $(0.08,0.89,0.02)$ \\
$x_{2}$ & $(0.73,0.12,0.08)$ & $(0.13,0.64,0.21)$ & $(0.03,0.82,0.13)$ & $(0.73,0.15,0.08)$ \\
$x_{3}$ & $(0.91,0.03,0.02)$ & $(0.07,0.09,0.05)$ & $(0.04,0.85,0.10)$ & $(0.68,0.26,0.06)$ \\
$x_{4}$ & $(0.85,0.09,0.05)$ & $(0.74,0.16,0.10)$ & $(0.02,0.89,0.05)$ & $(0.08,0.84,0.06)$ \\
$x_{5}$ & $(0.90,0.05,0.02)$ & $(0.68,0.08,0.21)$ & $(0.05,0.87,0.06)$ & $(0.13,0.75,0.09)$ \\
\hline
\end{tabular}

PFWMM operator

or the PFWDMM operator

$$
\alpha_{i}=P F W M M^{P}\left(\alpha_{i 1}, \alpha_{i 2}, \ldots, \alpha_{i n}\right),
$$

$$
\alpha_{i}=P F W D M M^{P}\left(\alpha_{i 1}, \alpha_{i 2}, \ldots, \alpha_{i n}\right),
$$

to aggregate attribute values, so that a series of comprehensive preference value can be obtained.

\subsection{Decision making process}

Step 1. As all of the attribute values are the same type, the original decision matrix does not need to be standardized.

Step 2. For each alternative, we utilize the PFWMM operator to aggregate decision makers' 
preference information. Therefore, a series of overall assessments can be obtained. Here we assume $P=(1,1,1,1)$, then the overall values of alternative are

$$
\begin{aligned}
& \alpha_{1}=(0.8460,0.1137,0.0258), \\
& \alpha_{2}=(0.8086,0.1176,0.0476), \\
& \alpha_{3}=(0.8488,0.0622,0.0234), \\
& \alpha_{4}=(0.8358,0.1214,0.0283), \\
& \alpha_{5}=(0.8477,0.0867,0.0308) .
\end{aligned}
$$

Step 3. Based on Definition 3, we can calculate the score function $S\left(\alpha_{i}\right)(i=1,2,3,4,5)$ as follows $S\left(\alpha_{1}\right)=0.8202, S\left(\alpha_{2}\right)=0.7610, S\left(\alpha_{3}\right)=0.8254$, $S\left(\alpha_{4}\right)=0.8075, S\left(\alpha_{5}\right)=0.8169$.

Step 4. According to the ranking order of the overall values, we can get the ranking order of the corresponding alternatives.

That is $\alpha_{3}>\alpha_{1}>\alpha_{5}>\alpha_{4}>\alpha_{2}$. Therefore $A_{3}$ is the best alternative.

\begin{tabular}{|c|c|c|}
\hline Parameter vector $P$ & The score function $s\left(\alpha_{i}\right)(i=1,2,3,4,5)$ & Ranking results \\
\hline \multirow{2}{*}{$P=(1,0,0,0)$} & $S\left(\alpha_{1}\right)=0.4129 \quad S\left(\alpha_{2}\right)=0.2503$ & \multirow{2}{*}{$A_{3} \mathrm{f} A_{1} \mathrm{f} A_{5} \mathrm{f} A_{4} \mathrm{f} A_{2}$} \\
\hline & $S\left(\alpha_{3}\right)=0.4288 \quad S\left(\alpha_{4}\right)=0.3796 S\left(\alpha_{5}\right)=0.3986$ & \\
\hline \multirow{2}{*}{$P=(1,1,0,0)$} & $S\left(\alpha_{1}\right)=0.6647 \quad S\left(\alpha_{2}\right)=0.5609$ & \multirow{2}{*}{$A_{3}$ f $A_{1}$ f $A_{5}$ f $A_{4}$ f $A_{2}$} \\
\hline & $S\left(\alpha_{3}\right)=0.6743 \quad S\left(\alpha_{4}\right)=0.6428 \quad S\left(\alpha_{5}\right)=0.6579$ & \\
\hline \multirow{2}{*}{$P=(1,1,1,0)$} & $S\left(\alpha_{1}\right)=0.7659 \quad S\left(\alpha_{2}\right)=0.6904$ & \multirow{2}{*}{$A_{3}$ f $A_{1}$ f $A_{5}$ f $A_{4}$ f $A_{2}$} \\
\hline & $S\left(\alpha_{3}\right)=0.7726 \quad S\left(\alpha_{4}\right)=0.7498 \quad S\left(\alpha_{5}\right)=0.7614$ & \\
\hline \multirow{2}{*}{$P=(1,1,1,1)$} & $S\left(\alpha_{1}\right)=0.8202 \quad S\left(\alpha_{2}\right)=0.7610$ & \multirow{2}{*}{$A_{3}$ f $A_{1}$ f $A_{5}$ f $A_{4}$ f $A_{2}$} \\
\hline & $S\left(\alpha_{3}\right)=0.8254 \quad S\left(\alpha_{4}\right)=0.8075 S\left(\alpha_{5}\right)=0.8169$ & \\
\hline \multirow{2}{*}{$P=(0.25,0.25,0.25,0.25)$} & $S\left(\alpha_{1}\right)=0.9738 \quad S\left(\alpha_{2}\right)=0.7208$ & \multirow{2}{*}{$A_{3} \mathrm{f} A_{1} \mathrm{f} A_{4} \mathrm{f} A_{5} \mathrm{f} A$} \\
\hline & $S\left(\alpha_{3}\right)=0.9765 \quad S\left(\alpha_{4}\right)=0.9717 \quad S\left(\alpha_{5}\right)=0.9687$ & \\
\hline \multirow{2}{*}{$P=(2,0,0,0)$} & $S\left(\alpha_{1}\right)=0.4659 \quad S\left(\alpha_{2}\right)=0.3155$ & \multirow{2}{*}{$A_{3} \mathrm{f} A_{5} \mathrm{f} A_{1} \mathrm{f} A_{4} \mathrm{f} A_{2}$} \\
\hline & $S\left(\alpha_{3}\right)=0.5063 \quad S\left(\alpha_{4}\right)=0.4534 \quad S\left(\alpha_{5}\right)=0.4708$ & \\
\hline \multirow{2}{*}{$P=(3,0,0,0)$} & $S\left(\alpha_{1}\right)=0.5060 \quad S\left(\alpha_{2}\right)=0.3541$ & \multirow{2}{*}{$A_{3} \mathrm{f} A_{5} \mathrm{f} A_{1} \mathrm{f} A_{4} \mathrm{f} A_{2}$} \\
\hline & $S\left(\alpha_{3}\right)=0.5510 \quad S\left(\alpha_{4}\right)=0.4940 S\left(\alpha_{5}\right)=0.5157$ & \\
\hline
\end{tabular}

In step 3, if we utilize the PFWDMM operator to aggregate decision makers' assessments, then the overall values of alternatives are

Table $2.5 c$

Table 3. Score functions and ranking results by using the different parameter vector $P$ in the PFWDMM operator

$$
\begin{aligned}
& \alpha_{1}=(0.1336,0.8255,0.5076), \\
& \alpha_{2}=(0.0823,0.8209,0.5661), \\
& \alpha_{3}=(0.0790,0.7808,0.4647), \\
& \alpha_{4}=(0.0699,0.8718,0.4788), \\
& \alpha_{5}=(0.0956,0.8448,0.5304) .
\end{aligned}
$$

Therefore, the scores of overall values are

$$
\begin{aligned}
& S\left(\alpha_{1}\right)=-0.3740, S\left(\alpha_{2}\right)=-0.4838, \\
& S\left(\alpha_{3}\right)=-0.3857, S\left(\alpha_{4}\right)=-0.4089, \\
& S\left(\alpha_{5}\right)=-0.4347 .
\end{aligned}
$$

Then the ranking result is $A_{1} \mathrm{f} A_{3} \mathrm{f} A_{4} \mathrm{f} A_{5} \mathrm{f} A_{2}$.

\subsection{The influence of the parameter vector $P$ on the ranking results}

In this section, we investigate the influence of the parameter vector $P$ on the final ranking results. We assign different values to $P$ and the scores functions and ranking ordered are presented in Tables 2 and 3. 


\begin{tabular}{|c|c|c|}
\hline Parameter vector $P$ & The score function $s\left(\alpha_{i}\right)(i=1,2,3,4,5)$ & Ranking results \\
\hline \multirow{2}{*}{$P=(1,0,0,0)$} & $S\left(\alpha_{1}\right)=0.3701 S\left(\alpha_{2}\right)=0.1881$ & \multirow{2}{*}{$A_{1} \mathrm{f} A_{5} \mathrm{f} A_{3} \mathrm{f} A_{4} \mathrm{f} A_{2}$} \\
\hline & $S\left(\alpha_{3}\right)=0.2338 \quad S\left(\alpha_{4}\right)=0.1990 \quad S\left(\alpha_{5}\right)=0.2520$. & \\
\hline$P=(1,1,0,0)$ & $\begin{array}{c}S\left(\alpha_{1}\right)=-0.0083 \quad S\left(\alpha_{2}\right)=-0.1626 \\
S\left(\alpha_{3}\right)=-0.0642 \quad S\left(\alpha_{4}\right)=-0.0944 \quad S\left(\alpha_{5}\right)=-0.0992\end{array}$ & $A_{1} \mathrm{f} A_{3} \mathrm{f} A_{4} \mathrm{f} A_{5} \mathrm{f} A_{2}$ \\
\hline$P=(1,1,1,0)$ & $\begin{array}{c}S\left(\alpha_{1}\right)=-0.2309 \quad S\left(\alpha_{2}\right)=-0.3601 \\
S\left(p_{3}\right)=-0.2560 \quad S\left(\alpha_{4}\right)=-0.2825 \quad S\left(\alpha_{5}\right)=-0.3039\end{array}$ & $A_{1} \mathrm{f} A_{3} \mathrm{f} A_{4} \mathrm{f} A_{5} \mathrm{f} A_{2}$ \\
\hline$P=(1,1,1,1)$ & $\begin{array}{c}S\left(\alpha_{1}\right)=-0.3740 \quad S\left(\alpha_{2}\right)=-0.4838 \\
S\left(\alpha_{3}\right)=-0.3857 \quad S\left(\alpha_{4}\right)=-0.4089 \quad S\left(\alpha_{5}\right)=-0.4347\end{array}$ & $A_{1} \mathrm{f} A_{3} \mathrm{f} A_{4} \mathrm{f} A_{5} \mathrm{f} A_{2}$ \\
\hline$P=(0.25,0.25,0.25,0.25)$ & $\begin{array}{c}S\left(\alpha_{1}\right)=-0.3320 \quad S\left(\alpha_{2}\right)=-0.4647 \\
S\left(\alpha_{3}\right)=-0.3596 \quad S\left(\alpha_{4}\right)=-0.3912 \quad S\left(\alpha_{5}\right)=-0.3948\end{array}$ & $A_{1}$ f $A_{3}$ f $A_{4}$ f $A_{5}$ f $A_{2}$ \\
\hline$P=(2,0,0,0)$ & $\begin{array}{c}S\left(\alpha_{1}\right)=0.3062 \quad S\left(\alpha_{2}\right)=0.1406 \\
S\left(\alpha_{3}\right)=0.1849 \quad S\left(\alpha_{4}\right)=0.1548 \quad S\left(\alpha_{5}\right)=0.1872\end{array}$ & $A_{1}$ f $A_{5}$ f $A_{3}$ f $A_{4}$ f $A_{2}$ \\
\hline$P=(3,0,0,0)$ & $\begin{array}{c}S\left(\alpha_{1}\right)=0.2576 \quad S\left(\alpha_{2}\right)=0.1042 \\
S\left(\alpha_{3}\right)=0.1534 \quad S\left(\alpha_{4}\right)=0.1257 \quad S\left(\alpha_{5}\right)=0.1423\end{array}$ & $A_{1} \mathrm{f} A_{3} \mathrm{f} A_{5} \mathrm{f} A_{4} \mathrm{f} A_{2}$ \\
\hline
\end{tabular}

As seen in Tables 2 and 3, different scores of the overall assessments and the ranking results can be obtained with regarding of the parameter vector $P$. However, although the ranking results are different, the best alternatives are always the same. For the PFWMM operator, the best alternatives is always $A_{3}$ and for the PFWDMM operator, the best alternatives is always $A_{l}$. In addition, for the PFWMM operator, the more interrelationship among PFNs are taken into consideration, the greater the scores of the overall values will become. However, the PFWDMM operator is opposite, which means that the more interrelationship among PFNs are captured, the smaller the cores of the overall values will become. Therefore, different parameter vector $P$ can be regarded as the decision makers' risk preference.

\subsection{Comparative analysis}

To illustrate the superiorities of the proposed method, we conduct comparative analysis. We compare the proposed method with Wei's [32] method based on picture fuzzy weighted averaging (PFWA) or picture fuzzy weighted geometric (PFWG) operators. We utilize Wei's [32] method and the proposed method in this paper to solve the above problem. The ranking results are shown in Table 4.

Table 4. Ranking results by using different methods

\begin{tabular}{c|c}
\hline Method & Ranking results \\
\hline $\begin{array}{c}\text { Wei's [32] method based on } \\
\text { PFWA operator }\end{array}$ & $A_{3} \mathrm{f} A_{2} \mathrm{f} A_{1} \mathrm{f} A_{5} \mathrm{f} A_{4}$ \\
\hline $\begin{array}{l}\text { Wei's [32] method based on } \\
\text { the PFWG [32] operator }\end{array}$ & $A_{3} \mathrm{f} A_{1} \mathrm{f} A_{2} \mathrm{f} A_{5} \mathrm{f} A_{4}$ \\
\hline
\end{tabular}

\begin{tabular}{c|c}
\hline $\begin{array}{c}\text { The proposed method based } \\
\text { on the PFWMM operator } \\
(P=(1,1,1,1))\end{array}$ & $A_{3} \mathrm{f} A_{1} \mathrm{f} A_{5} \mathrm{f} A_{4} \mathrm{f} A_{2}$ \\
\hline $\begin{array}{c}\text { The proposed method based } \\
\text { on the PFWDMM operator } \\
(P=(1,1,1,1))\end{array}$ & $A_{1} \mathrm{f} A_{3} \mathrm{f} A_{4} \mathrm{f} A_{5} \mathrm{f} A_{2}$ \\
\hline
\end{tabular}

From Table 4, we can find that the ranking results derived by Wei's method are different from the ranking orders obtained by the proposed method in this paper. The reason why the ranking results by using the PFWA operator and the PFWMM operator are different is that the former operator cannot consider the interrelationship between PFNs and the latter operator can capture the interrelationship among all PFNs. The reason why the ranking results by utilizing the PFWG and the PFWDMM operators are different is similar. Additionally, the proposed method in the paper is more general and flexible. When $P=(1,0, \ldots, 0)$ in the PFWMM operator, then proposed method is reduced to the Wei's [32] method based on PFWA operator. When $P=(1,0, \ldots, 0)$ in the PFWDMM operator, then proposed method is reduced to the Wei's [32] method based on PFWG operator. In other words, Wei's [32] method is special case of our method. Therefore, the proposed method is more powerful and general than Wei's [32] method. 


\section{Conclusion}

The recently proposed PFS has higher capacity than IFS for handling fuzziness and uncertainty in the process of MADM. The main contributions of this paper are that a series of picture fuzzy aggregation operators as well as a novel method to picture fuzzy MADM problems were proposed. More specifically, we extended traditional MM and DMM to picture fuzzy environment and proposed PFMM, PFWMM, PFDMM, and PFWDMM operators. These operators can reflect the correlations among all the picture fuzzy elements. Moreover, these operators make the information aggregation flexible as they have a vector of parameters. Some existing aggregation operators are special cases of the proposed operators. Furthermore, we propose a novel approach to MADM with picture fuzzy information. Finally, we applied the proposed method in a real decision making problem to illustrate the effectiveness of the proposed method. To better demonstrate the advantages of the proposed method, we compare it with other methods. In future works, we will investigate more aggregation operators for aggregating PFNs. For example, due to time shortage or a lack of expertise, decision makers may provide unduly high or low assessment values which negatively affect the decision results. Thus, reducing or eliminating such negative influence should be considered when fusing decision makers' ideas. In addition, considering $\mathrm{MM}$ is an effective and powerful aggregation technology, we will unitize it to fuse more fuzz information, such as hesitant picture fuzzy information, interval-valued picture fuzzy information, $q$-rung orthopair fuzzy information [49], $q$-rung interval-valued orthopair fuzzy information, $q$-rung orthopair linguistic information and so forth.

\section{Acknowledgements}

This work was partially supported by National Science Foundation of China (Grant number 61702023, 71532002), Humanities and Social Science Foundation of Ministry of Education of China (Grant number 17YJC870015).

\section{References}

[1] L.A. Zadeh, Fuzzy sets, Information and Control 8 (1965), 338-353.
[2] J.M. Merigó, Fuzzy decision making using immediate probabilities, Computers \& Industrial Engineering 58 (2010), 651657.

[3] J.M. Merigó, A.M. Gil-Lafuente, and R.R. Yager, An overview of fuzzy research with bibliometric indicators, Applied Soft Computing 27 (2015), 420-433.

[4] F. Blanco-Mesa, J.M. Merigó, and A.M. Gil-Lafuente, Fuzzy decision making: A bibliometric-based review, Journal of Intelligent \& Fuzzy Systems 32 (2017), 2033-2050.

[5] K.T. Atanassov, Intuitionistic fuzzy sets, Fuzzy Sets and Systems 33 (1989), 37-46.

[6] Z.S. Xu, Intuitionistic fuzzy aggregation operators, IEEE Transactions on fuzzy systems 15 (2007), 1179-1187.

[7] J. Qin and X. Liu, An approach to intuitionistic fuzzy multiple attribute decision making based on Maclaurin symmetric mean operators, Journal of Intelligent and Fuzzy Systems $\mathbf{2 7}$ (2014), 2177-2190.

[8] J. Guo, A risk assessment approach for failure mode and effects analysis based on intuitionistic fuzzy sets and evidence theory, Journal of Intelligent and Fuzzy Systems 30 (2016), 869-881.

[9] Q. Cao, J. Wu, and C. Liang, An intuitionistic fuzzy judgement matrix and TOPSIS integrated multi-criteria decision making method for green supplier selection, Journal of Intelligent and Fuzzy Systems 28 (2015), 117-126.

[10] S.P. Wang, F. Wang, and J.Y. Dong, A three-phase method for group decision making with interval-valued intuitionistic fuzzy preference relations, IEEE Transactions on Fuzzy Systems 26 (2018), 998-1010

[11]C.Y. Wang and S.M. Chen, A new multiple attribute decision making method based on linear programming methodology and novel score function and novel accuracy function of interval-valued intuitionistic fuzzy values, Information Sciences 438 (2018), 145-155.

[12]W. Jiang, B.Y. Wei, X. X.Y. Li, and H.Q. Zheng, Intuitionistic fuzzy power aggregation operator based on entropy and its application in decision making, International Journal of Intelligent Systems 33 (2018), 49-67.

[13]Z.M. Zhang, Multi-criteria group decision-making methods based on new intuitionistic fuzzy Einstein hybrid weighted aggregation operators, Neural Computing and Applications 28 (2017), 3781-3800.

[14]P.D. Liu, S.M. Chen and J.L. Liu, Multiple attribute group decision making based on intuitionistic fuzzy interaction partitioned Bonferroni mean operators, Information Sciences 411 (2017), 98-121.

[15]F. Meng and C. Tan, A method for multi-attribute group decision making based on generalized interval-valued intuitionistic fuzzy Choquet integral operators, International Journal of Uncertainty, Fuzziness and Knowledge-Based Systems $\mathbf{2 5}$ (2017), 821-849.

[16]J. Ye, Intuitionistic fuzzy hybrid arithmetic and geometric aggregation operators for the decision-making of mechanical design schemes, Applied Intelligence 47 (2017), 743-751.

[17]Z.L. Yang, J.Q. Li, L.C. Huang, and Y.Y. Shi, Developing dynamic intuitionistic normal fuzzy aggregation operators for multi-attribute decision-making with time sequence preference, Expert Systems with Applications 82 (2017), 344-356.

[18]P.D. Liu and S.M. Chen, Group decision making based on Heronian aggregation operators of intuitionistic fuzzy numbers, IEEE transactions on cybernetics 47 (2017), 2514-2530.

[19] S. Das, D. Guha, and R. Mesiar, Extended Bonferroni Mean Under Intuitionistic Fuzzy Environment Based on a Strict tConorm, IEEE Transactions on Systems, Man, and Cybernetics: Systems 47 (2017), 2083-2099. 
[20]P.D. Liu and H.G. Li, Interval-valued intuitionistic fuzzy power Bonferroni aggregation operators and their application to group decision making, Cognitive Computation 9 (2017), 494-512.

[21]C.Y. Wang and S.M. Chen, A new multiple attribute decision making method based on linear programming methodology and novel score function and novel accuracy function of interval-valued intuitionistic fuzzy values, Information Sciences 438 (2018), 145-155.

[22] Y.N. Wang, Y.P. Dai, Y.W. Chen, and F.C. Meng, The evidential reasoning approach to medical diagnosis using intuitionistic fuzzy Dempster-Shafer theory, International Journal of Computational Intelligence Systems 8 (2015), 75-94.

[23]Z. Wang, Z.S. Xu, S.S. Li, and Z.Q. Yao, Direct clustering analysis based on intuitionistic fuzzy implication, Applied Soft Computing 23 (2014), 1-8.

[24]Z. Wang, Z.S. Xu, S.S. Liu, and J. Tang, A netting clustering analysis method under intuitionistic fuzzy environment, $A p$ plied Soft Computing 11 (2011), 5558-5564.

[25] S.M. Chen, S.H. Cheng, and T.C. Lan, A novel similarity measure between intuitionistic fuzzy sets based on the centroid points of transformed fuzzy numbers with applications to pattern recognition, Information Sciences 343 (2016), 15-40.

[26] C.M. Hwang, M.S. Yang, W.L. Huang, and M.G. Lee, A similarity measure of intuitionistic fuzzy sets based on the Sugeno integral with its application to pattern recognition, Information Sciences 189 (2012), 93-109.

[27]B. Cuong, Picture fuzzy sets-first results. part 1, in: Seminar Neuro-Fuzzy systems with applications, Institute of Mathematics 4 (2013)

[28]L.H. Son, P.V. Viet and P.V. Hai, Picture inference system: a new fuzzy inference system on picture fuzzy set, Applied Intelligence 46 (2017), 652-669.

[29] P.H. Thong, Some novel hybrid forecast methods based on picture fuzzy clustering for weather nowcasting from satellite image sequences, Applied Intelligence 46 (2017), 1-15.

[30]G.W. Wei, Some similarity measures for picture fuzzy sets and their applications, Iranian Journal of Fuzzy Systems 15 (2018), 77-89.

[31]H. Garg, Some Picture fuzzy aggregation operators and their applications to multicriteria decision-making, Arabian Journal for Science and Engineering 8 (2107), 1-16.

[32] G. Wei, Picture fuzzy aggregation operators and their application to multiple attribute decision making, Journal of Intelligent and Fuzzy Systems 33 (2017), 713-724.

[33]C. Bonferroni, Sulle medie multiple di potenze, Bolletino Matematica Italiana 5 (1950), 267-270.

[34] S. Sykora, Mathematical Means and Averages: Generalized Heronian Means, Stan's Library (2009).

[35]Z.S. Xu and R.R. Yager, Intuitionistic fuzzy Bonferroni means, IEEE Transactions on Systems, Man, and Cybernetics, Part B (Cybernetics) 41 (2011), 568-578.

[36] M.M. Xia, Z.S. Xu, and B. Zhu, Geometric Bonferroni means with their application in multi-criteria decision making, Knowledge-Based Systems 40 (2013), 88-100.

[37]P.D. Liu and S.M. Chen, Group decision making based on Heronian aggregation operators of intuitionistic fuzzy numbers, IEEE transactions on cybernetics 47 (2017), 2514-2530.

[38].J. Yu, Intuitionistic fuzzy geometric Heronian mean aggregation operators, Applied Soft Computing 13 (2013), 1235-1246.

[39]D.J. Yu, D.F. Li, and J.M. Merigó, Dual hesitant fuzzy group decision making method and its application to supplier selection, International Journal of Machine Learning and Cybernetics 7 (2016), 819-831.

[40]H.N. Tu, C.Y. Wang, X.Q. Zhou, and S.D. Tao, Dual hesitant fuzzy aggregation operators based on Bonferroni means and their applications to multiple attribute decision making, Annals of Fuzzy Mathematics and Informatics 14 (2017), 265278.

[41]C. Maclaurin, A second letter to Martin Folkes, Esq.; concerning the roots of equations, with the demonstration of other rules in algebra, Phil, Transaction 36 (1729), 59-96

[42]R.F. Muirhead, Some methods applicable to identities and inequalities of symmetric algebraic functions of n letters, Proceedings of the Edinburgh Mathematical Society 21 (1902), 144-162.

[43]J.D. Qin and X.W. Liu, 2-tuple linguistic Muirhead mean operators for multiple attribute group decision making and its application to supplier selection, Kybernetes 45 (2016), 2-29.

[44]P.D. Liu and D.F. Li, Some Muirhead mean operators for intuitionistic fuzzy numbers and their applications to group decision making, PloS one 12 (2017), Article ID e0168767.

[45]L. Li, R.T. Zhang, J. Wang, X.M. Zhu, and Y.P. Xing, Pythagorean fuzzy power Muirhead mean operators with their application to multi-attribute group decision making, Journal of Intelligent and Fuzzy Systems, to be published.

[46] P.D. Liu, Y. Li, M.C. Zhang, L. Zhang, and J. Zhao, Multipleattribute decision-making method based on hesitant fuzzy linguistic Muirhead mean aggregation operators, Soft Computing (2018), 1-12

[47]J. Wang, R.T. Zhang, X.M. Zhu, Y.P. Xing, and B. Buchmeister, Some hesitant fuzzy linguistic Muirhead means with their application to multi-attribute group decision making, Complexity, to be published.

[48] X.W. Liao, Y. Li, and B. Lu, A model for selecting an ERP system based on linguistic information process, Information Systems 32 (2007), 1005-1017.

[49] R.R. Yager, Generalized orthopair fuzzy Sets, IEEE Transactions on Fuzzy Systems 25 (2017), 1222-1230. 